\title{
MÉTODO DE ESCOLHA DE MEDICAMENTOS ANTI-HIPERTENSIVOS POR GESTORES DA ÁREA DE SAÚDE
}

\author{
Rondinelli de Carvalho LADEIRA ${ }^{1}$, Milton ERTHAL JÚNIOR ${ }^{2,3}$ \& Henrique Rego Monteiro DA HORA ${ }^{2,3 *}$ \\ ${ }^{1}$ Universidade Iguaçu - Campus V, Itaperuna, Rio de Janeiro, Brasil. \\ ${ }^{2}$ Instituto Federal Fluminense - Campos dos Goytacazes, Rio de Janeiro, Brasil. \\ ${ }^{3}$ Universidade Candido Mendes - Mestrado em Pesquisa Operacional e Inteligência Computacional, Campos do \\ Goytacazes, Rio de Janeiro, Brasil. \\ *Autor para correspondência: dahora@gmail.com
}

http://dx.doi.org/10.18571/acbm.098

\section{RESUMO}

Um problema enfrentado pelos gestores de saúde é a aquisição de medicamentos que, para o abastecimento do SUS, deve-se levar em consideração a efetividade, os custos e a segurança dos mesmos. A análise farmacoeconômica pode ser vista como a descrição e a análise dos custos da terapia farmacêutica para os sistemas de assistência à saúde e para a sociedade. O presente trabalho faz a análise de custo-efetividade de cinco betabloqueadores adrenérgicos usados no tratamento de hipertensão arterial (atenolol, carvedilol, metoprolol, propranolol e sotalol) através de auxílio multicritério à decisão. Tendo em vista gestores de saúde como agentes da decisão, foram testados os métodos de Borda para definição dos pesos dos critérios por especialistas e AHP para escolha do medicamento através do software IPE 1.0. Questionários foram aplicados a sete profissionais médicos especialistas em cardiologia para a comparação dos critérios (efetividade, custo, experiência com o fármaco e segurança), sub-critérios (agravamento da insuficiência cardíaca congestiva, agravamento da doença arterial periférica, broncoespasmo, bradicardia e alterações metabólicas, relativos ao critério segurança) e alternativas à luz dos critérios estudados. O betabloqueador que se apresentou como melhor escolha foi o atenolol $(32,38 \%)$.

Palavras-chave: Tomada de decisões; Hipertensão; Gestão em Saúde.

\begin{abstract}
A problem faced by health managers is purchasing medicines, to supply the SUS, should take into account the effectiveness, cost and safety. A pharmacoeconomic analysis can be seen as the description and analysis of the costs of drug therapy to health care systems and society. This paper makes the cost-effectiveness of five adrenergic blockers used to treat hypertension (atenolol, carvedilol, metoprolol, propranolol and sotalol) through multicriteria decision aid. Considering health managers as agents of the decision, were tested methods for defining the edge weights of the criteria by experts and AHP for choice of medication through the IPE 1.0 software. Questionnaires were administered to seven professional specialists in cardiology for comparison of criteria (effectiveness, cost, experience with the drug and safety), sub-criteria (worsening of congestive heart failure, worsening of peripheral arterial disease, bronchospasm, bradycardia and metabolic changes relating to criterion security), and alternatives in the light of the criteria studied. The beta-adrenergic blocker who introduced himself as best choice was atenolol $(32.38 \%)$.
\end{abstract}

Keywords: Decision Making; Hypertension; Health Management. 


\section{Introdução}

Nem sempre é possível tomar uma decisão acertada em uma organização (GOMES e Gomes, 2012). Com exceção de problemas rotineiros, bem conhecidos e com estrutura de opções bem definida, os demais problemas de decisão não estão estruturados, sem alternativas de ação e critérios claros sendo que a escolha da melhor opção quase sempre é caótica e complexa. Isso ocorre, pois os indivíduos e as organizações não possuem visão clara e completa dos objetivos e dos meios que definem o problema de decisão. Incertezas, a falta de estruturação e do tamanho do problema pode inviabilizar a aplicação sistemática das metodologias de decisão, principalmente daquelas que utilizam julgamentos subjetivos.

O auxílio multicritério à decisão possui uma abordagem essencialmente interdisciplinar. Não apenas nos recursos que utiliza, mas no universo de problemas que se dispõe a ajudar a resolver. Esta metodologia é útil a homens de negócios, gestores públicos, engenheiros e profissionais de áreas afins, dentro e fora da academia. Seu enfoque analítico é pertinente na atual conjuntura de complexidade crescente, de informação abundante e de maior abertura a temas que eram distantes das corporações privadas (GOMES e GOMES, 2012).

Vários autores têm discutido a utilização de ferramentas de AMD em uma ampla variedade de aplicações. As áreas de engenharia e ciências da computação são as mais estudadas, enquanto que a área médica apresenta menos de 5\% do total das publicações (MENDES e ERTHAL JÚNIOR, 2012). Um levantamento bibliográfico (LIBERATORE e NYDICK, 2008) analisou 50 artigos específicos sobre AHP na área de saúde e a tabela 1 classifica-os de acordo com sua categoria.

Tabela 1: Artigos da área médica publicados por categoria utilizando AHP nas bases Pub Med, CINAL e PsycINFO.

\begin{tabular}{lc}
\hline CATEGORIA DA SAÚDE & TOTAL DE TRABALHOS \\
\hline Diagnóstico & 04 \\
Participação do paciente & 08 \\
Terapia/Tratamento & 08 \\
Transplante de órgãos & 04 \\
Projeto e tecnologia de avaliação e seleção & 14 \\
Planejamento de recursos humanos & 03 \\
Avaliação de saúde e política & 09 \\
\hline
\end{tabular}

A Lei ${ }^{\circ} 8080 / 90$, regulamentada pelo decreto $n^{\circ} 7.508$ de 28 de junho de 2011, estabelece que estejam incluídas no campo de atuação do SUS a assistência terapêutica integral, inclusive a farmacêutica. Para cumprir esta exigência legal o estado brasileiro tem elevado os gastos com saúde ano após ano. O maior número de idosos e o aumento das doenças crônico-degenerativas foram responsáveis pelo aumento nos gastos públicos com saúde, que representaram 7\% do PIB, dos quais mais da metade se referem ao setor privado (PIOLA e VIANNA, 2009).

Com o intuito de aumentar e qualificar o acesso da população brasileira aos medicamentos, o Ministério da Saúde criou em 1998 a Política Nacional de Medicamentos (PNM), que é um elemento fundamental para implementar ações que promovam a melhoria das condições da assistência à saúde. Uma das diretrizes do PNM é a adoção da Relação Nacional de Medicamentos (RENAME) que contempla os medicamentos essenciais cuja lista segundo a Organização Mundial de Saúde (OMS). Medicamentos essenciais são os que satisfazem às necessidades prioritárias de saúde da população. São selecionados por critério de relevância na saúde pública, pela segurança, eficácia e sua relação custo efetividade (WHO, 2016).

A análise farmacoeconômica é a descrição e análise dos custos da terapia farmacêutica para os sistemas de assistência à saúde e para a sociedade. Quanto às intervenções farmacêuticas, a farmacoeconomia tenta medir se o benefício adicionado por uma intervenção compensa o custo adicionado por essa intervenção. A Figura 1 descreve uma equação farmacoeconômica básica, 
onde se comparam os custos da adição de um produto ou serviço farmacêutico $\left(\mathrm{R}_{\mathrm{X}}\right)$ aos desfechos (CORDEIRO e LEITE, 2005; RASCATI, 2009).

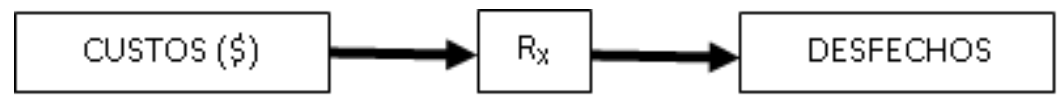

Figura 1: Equação farmacoeconômica básica (Rascati, 2009).

Existem quatro tipos básicos de estudos farmacoeconômicos: análise de minimização de custos; análise de custo-efetividade; análise de custo-utilidade; e análise de custo-benefício (RASCATI, 2009; SECOLI et al., 2005; TONON, TOMO e SECOLI, 2008). Todas as análises avaliam custos, mas diferem quanto aos resultados na saúde da população. A análise de custoefetividade é a mais utilizada por ser semelhante à prática cotidiana e utilizar as mesmas unidades dos ensaios clínicos (RASCATi, 2009; SECOLI et al., 2005).

Para cumprir esta lei, o Estado Brasileiro, nas esferas federal, estadual e municipal, precisa adquirir fármacos essenciais à população. A compra destes fármacos deve cumprir a lei de licitação pública (Lei no 8.666 de 1993), que segue o princípio do menor preço para aquisição do fármaco, entre eles os betabloqueadores, usados para regular a pressão arterial.

Os betabloqueadores se diferenciam de acordo com o princípio ativo e podem causar efeitos colaterais diversos, além de ação sinérgica com outros medicamentos. Muitas vezes, os betabloqueadores de baixo custo causam efeitos colaterais que provocam o retorno dos pacientes aos postos de saúde, colaborando com a superlotação das clinicas e hospitais públicos, e elevando os gastos totais no tratamento dos pacientes afetados. Desta forma, faz-se necessário o uso de técnicas e pesquisas específicas para avaliar a classe de medicamentos mais efetiva e de baixo custo para a população.

Segundo dados do Ministério da Saúde foram gastos, em 2008, R\$ 6 bilhões com internações, exceto partos, sendo que deste montante $58 \%$ ( $\mathrm{R} \$ 3,5$ bilhões) foram exclusivos para atendimento de doenças crônicas, principalmente as doenças cardiovasculares ( $\mathrm{R} \$ 770$ milhões) (SILVA e FERREIRA, 2011). A American Heart Association ressalta que embora se tenha conseguido enorme progresso na luta contra as doenças cardiovasculares, desde 1900, essas doenças têm sido a principal causa de morte entre norte-americanos (SILVA e FERREIRA, 2011).

O crescimento da população de idosos, os maus hábitos alimentares e a falta de exercícios físicos aumentarão, nas próximas décadas, o número de doenças crônicas degenerativas do coração. Consequentemente, espera-se elevação dos gastos com tratamentos intra-hospitalares, ambulatoriais e seguros sociais por conta destas doenças (CAMPOS e PORTO, 2012).

Nos últimos tempos ocorreram grandes avanços na área de tratamento de doenças coronarianas, inclusive entre os betabloqueadores, que aumentaram a sobrevida de pacientes hipertensivos (VANZELLI et al., 2010). Segundo a Sociedade Brasileira de Hipertensão, a hipertensão atinge cerca de $25 \%$ da população brasileira adulta, chegando a $50 \%$ após os 60 anos, sendo responsável por $40 \%$ dos infartos, $80 \%$ dos derrames e $25 \%$ dos casos de insuficiência renal terminal. Porém, tais consequências podem ser evitadas com o tratamento adequado (GOMES et al., 2004).

Apesar de ser conhecida a importância do acesso da população aos medicamentos essenciais, pesquisas têm demonstrado que o SUS tem falhado na garantia de direito ao acesso gratuito aos medicamentos padronizados para o tratamento de hipertensão arterial e diabetes (VOSGERAU, CABRERA e SOUZA, 2011). O mesmo estudo observou ainda que a aquisição desses medicamentos em unidades básicas de saúde foi relativamente baixa $(54,7 \%)$ comparada a de outros estudos que identificaram taxa de 63,1\% em Porto Alegre (2009) e 93,9\% em Maringá (1999). 


\section{Biomedica Brasiliensia}

A seleção adequada e racional de fármacos é necessária tanto no âmbito hospitalar quanto na gestão do SUS, devido às inúmeras opções existentes no mercado, cerca de 3.500 princípios ativos e até 23.000 apresentações comerciais diferentes, e a uma necessidade de reduzir a um número apropriado dos medicamentos disponíveis para aquisição. Sendo então, necessária a utilização de métodos que minimizem a subjetividade e permitam tomadas de decisões de máxima eficiência, disponibilizando uma farmacoterapia de maior qualidade com menor custo (AMARAL e PROVIN, 2011).

Este trabalho tem como objetivo Avaliar a melhor opção de compra de medicamentos betabloqueadores adrenérgicos para uso como anti-hipertensivos utilizando métodos de auxílio à decisão.

O crescente aumento da expectativa de vida da população, mudanças nos hábitos alimentares e de vida, o estresse fazem com que haja um aumento do número de doenças crônico-degenerativas, incluindo as doenças cardiovasculares, como a hipertensão.

Com a criação da Política Nacional de Medicamentos em 1998, ampliou-se a oferta de medicamentos oferecidos à população. Por outro lado, a aquisição desses medicamentos tornouse motivo de preocupação pela evolução dos gastos. Dados do Ministério da Saúde mostram que enquanto os gastos com saúde aumentaram $9,6 \%$, os gastos com medicamentos aumentaram $123,9 \%$ no período entre 2002 a 2006 (VIEIRA, 2009).

O presente trabalho justifica-se devido a grande quantidade de opções de betabloqueadores no mercado, sendo que cada um apresenta um princípio ativo diferente, que geram efeitos colaterais diferenciados, o que torna difícil para os gestores da área de saúde tomarem uma decisão acertada durante o processo escolha na aquisição deste tipo de medicamento.

Ferramentas de auxílio à decisão minimizam a subjetividade na escolha permitindo aos decisores escolhas mais acertadas, disponibilizando fármacos de maior efetividade e segurança a custos mais baratos.

\subsection{Hipertensão Arterial e Betabloqueadores}

O coração é o responsável por fazer o sangue circular por todo o corpo. A força exercida por ele durante o bombeamento do sangue pelos vasos é chamada pressão arterial que é considerada normal, em média, para valores iguais ou inferiores a $120 \mathrm{mmHg}$ para pressão sistólica e $80 \mathrm{mmHg}$ para pressão diastólica (BRASIL, 2013; PAGE et al., 2004). O Ministério da Saúde (BRASIL, 2013) considera que a hipertensão arterial ocorre quando a medida da pressão se mantém frequentemente acima de 140 por $90 \mathrm{mmHg}$. O Quadro 1 traz a classificação da pressão sanguínea:

Quadro 1: Classificação da pressão sanguínea.

\begin{tabular}{|l|c|c|}
\hline Categoria & Sistólica $\mathbf{( m m H g )}$ & Diastólica (mmHg) \\
\hline Normal & $<130$ & $<85$ \\
\hline Normal elevada & $130-139$ & $85-89$ \\
\hline Hipertensão & & $90-99$ \\
\hline Estadiamento 1 (brando) & $140-159$ & $100-109$ \\
\hline Estadiamento 2 (moderado) & $160-179$ & $110-119$ \\
\hline Estadiamento 3 (grave) & $180-209$ & $\geq 120$ \\
\hline Estadiamento 4 (muito grave) & $\geq 210$ & \\
\hline
\end{tabular}

A Sociedade Brasileira de Cardiologia ressalta na VI Diretrizes de Hipertensão (GOMES et al., 2004) que a hipertensão arterial sistêmica (HAS) é um fator importante na morbimortalidade gerando risco de várias doenças. Por ser uma doença assintomática, os custos do tratamento e a falta de entendimento da patologia por muitos dos pacientes fazem com que eles procurem terapias não farmacológicas, como alívio do estresse e remédios caseiros (PUCCI et al., 2012). 
Os custos diretos anuais da sociedade brasileira em 2004 atribuídos a casos de doenças cardiovasculares graves superam os $\mathrm{R} \$ 11,2$ bilhões, distribuídos entre os setores públicos (47,3\%), saúde suplementar (18,8\%) e desembolso direto (33,9\%) (AZAMBUJA et al., 2008).

Na maioria dos pacientes com hipertensão, a pressão sanguínea se eleva progressivamente ao longo do tempo (PAGE et al., 2004). O tratamento passa a ser necessário para o resto da vida para se reduzir os riscos cardiovasculares ao longo de muitos anos.

O tratamento envolve medidas não farmacológicas, como a prática de exercícios físicos, redução no consumo de sal, de gorduras saturadas, de álcool, substituindo por frutas e fibras e a redução do peso corporal, seguidas pela introdução de fármacos, de forma gradativa (RANG, 2012). O início do tratamento com fármacos que tenham benefício comprovado e com menor probabilidade de gerar efeitos colaterais.

Os betabloqueadores adrenérgicos também chamados de antagonistas dos receptores $\beta$ adrenérgicos são recomendados como medicamentos de primeira escolha em hipertensão arterial. Este medicamento age reduzindo o débito cardíaco (WANNMACHER, 2007). Esta classe de fármaco tem recebido especial atenção clínica devido à sua eficácia no tratamento da hipertensão. Suas propriedades farmacológicas podem ser explicadas a partir das respostas produzidas por vários tecidos e pela atividade dos nervos simpáticos desses tecidos (BRUNTON, LAZO e PARKER, 2010).

Apesar do seu mecanismo de ação não estar totalmente elucidado (PAGE et al., 2004), atuam sobre os receptores $\beta$-adrenérgicos que se localizam predominantemente no coração, nas artérias e arteríolas do músculo esquelético e nos brônquios, bloqueando o estímulo cardíaco causando vasodilatação e broncoconstrição (KOROLKOVAS e FRANÇA, 2009).

Os betabloqueadores adrenérgicos inibem as resposta cronotrópicas, inotrópicas e vaso constritoras geradas pelas catecolaminas nos receptores beta-adrenérgicos, que são em número de três $\left(\beta_{1}, \beta_{2}\right.$ e $\left.\beta_{3}\right)$, todos ligados às proteínas G (HELFAND et al., 2009).

As ações dos receptores $\beta$ dependem da localização do tecido-alvo (BORTOLOTTO E CONSOLIM-COLOMBO, 2009), sendo que os receptores demonstrados no Quadro 2.

Quadro 2: Ações específicas dos receptores $\beta$.

\begin{tabular}{|c|c|c|}
\hline Receptores $\beta_{1}$ & Receptores $\boldsymbol{\beta}_{2}$ & Receptores $\boldsymbol{\beta}_{3}$ \\
\hline $\begin{array}{l}\text { - Aumento do débito cardíaco, por } \\
\text { aumento da frequência cardíaca e } \\
\text { do volume ejetado em cada } \\
\text { batimento; } \\
\text { - Liberação de renina nas células } \\
\text { justaglomerulares; } \\
\text { - Lipólise do tecido adiposo. }\end{array}$ & $\begin{array}{l}\text { - Relaxamento da musculatura lisa } \\
\text { (como nos brônquios, sistema } \\
\text { gastro-intestinal, urinário e útero } \\
\text { gravídico); } \\
\text { - Liberação de renina nos rins; } \\
\text { - Dilatação das artérias do músculo } \\
\text { esquelético; } \\
\text { - Lipólise do tecido adiposo; } \\
\text { - Glicogenólise e gliconeogênese; } \\
\text { - Inibição da liberação de } \\
\text { histamina nos mastócitos. }\end{array}$ & $\begin{array}{l}\text { - Predominantemente efeitos } \\
\text { metabólicos como a estimulação da } \\
\text { lipólise do tecido adiposo. }\end{array}$ \\
\hline
\end{tabular}

Esses fármacos são classificados conforme sua cardiosseletividade e atividade agonista parcial, o Quadro 3 demonstra essa classificação (KOROLKOVAS E FRANÇA, 2009). 
Quadro 3: Classificação dos fármacos antagonistas $\beta$-adrenoreceptores conforme a cardiosseletividade (Page et al., 2004). *possuem atividade $\beta 1$-agonista parcial.

\begin{tabular}{|c|c|c|}
\hline Cardiosseletivos & Não seletivos & $\begin{array}{c}\text { Drogas com atividade } \alpha 1 \\
\text { antagonista adicional }\end{array}$ \\
\hline Acebutolol* & Alprenolol* & Bucindolol \\
Atenolol & Carteolol* & Carvedilol \\
Betaxolol & Nadolol & Labetalol \\
Bevantolol & Oxprenolol* & \\
Bisoprolol & Penbutolol* & \\
Celiprolol & Pindolol* & \\
Esmolol & Propranolol & \\
Metoprolol & Sotalol & \\
Practolol* & Timolol & \\
\hline
\end{tabular}

Em relação à segurança dessa classe farmacológica, os principais efeitos adversos relatados surgem devido ao bloqueio dos receptores $\beta$; os efeitos adversos graves não relacionados com o bloqueio são raros (BRUNTON, LAZO e PARKER, 2010). Os principais efeitos adversos relacionados ao uso de betabloqueadores são (BRUNTON, LAZO e PARKER, 2010; KOROLKOVAS e FRANÇA, 2009; PAGE et al., 2004):

○ Agravamento da insuficiência cardíaca congestiva (ICC);

○ Agravamento da doença arterial periférica (DAP)

o Bradicardia;

o Broncoespasmo;

○ Alterações metabólicas.

A ICC pode ser induzida uma vez que o sistema nervoso simpático auxilia o desempenho cardíaco em pacientes com comprometimento da função do miocárdio, assim sendo, o bloqueio $\beta$-adrenérgico pode causar insuficiência cardíaca ou exacerbá-la em pacientes com insuficiência cardíaca compensada, com infarto agudo do miocárdio ou cardiomegalia (BRUNTON, LAZO e PARKER, 2010).

A farmacoterapia com betabloqueadores não deve ser mantida em pacientes que se queixam de extremidades frias durante o tratamento devido ao desenvolvimento do chamado fenômeno Raynaud (BRUNTON, LAZO e PARKER, 2010). Este problema é um tipo de doença arterial periférica (DAP) que foi caracterizada por como sendo uma palidez dos dedos durante vasoconstrição (RANG, 2012), seguida por cianose pela desoxigenação do sangue em estase e vermelhidão por hiperemia reativa após o retorno do fluxo sanguíneo. A vasoconstrição de pequenas artérias e arteríolas, que pode ser leve a intensa, causa ulcerações e gangrena nos dedos

Os betabloqueadores exercem efeitos importantes sobre o coração, porém os efeitos negativos inotrópicos e cronotrópicos são previsíveis. Pacientes com erros na condução atrioventricular, os antagonistas $\beta$-adrenérgicos podem causar bradiarritmias fatais, principalmente em pacientes que fazem uso concomitante de outros agentes antiarrítmicos, passíveis de afetar a função do nodo sinusal ou a condução atrioventricular (BRUNTON, LAZO e PARKER, 2010).

Os antagonistas $\beta$-adrenérgicos bloqueiam receptores $\beta_{2}$ na musculatura lisa dos brônquios que são responsáveis pela broncodilatação, portanto, tais medicamentos devem ser evitados em pacientes com asma. Antagonistas $\beta_{1}$-receptores possuem alguma vantagem, porém nenhum mostrou-se suficientemente específico para evitar completamente interações com adrenorreceptores $\beta_{2}$ (BRUNTON, LAZO e PARKER, 2010; PAGE et al., 2004).

Como os diuréticos, o uso de betabloqueadores está relacionado à piora do controle glicêmico e aumento na incidência de diabetes mellitus, devido à diminuição da captação muscular de glicose e aumento da resistência insulínica causada pela vasoconstrição periférica dos betabloqueadores (RANG, 2012). 


\section{Metodologia}

A metodologia proposta no presente trabalho visa criar um procedimento para auxiliar na escolha de medicamentos dentro de uma mesma classe terapêutica para aquisição dos mesmos por gestores da área da saúde. Como método decisório foram utilizados o método de Borda (COSTA, 2005) e o AHP (COSTA, 2006).

Este trabalho, dada a sua natureza, não se ocupará em descrever em detalhes os métodos de Borda e o AHP, uma vez que há na comunicação científica diversos trabalhos que se ocupam desta tarefa já que são dois métodos consolidados no arcabouço dos métodos multicritérios. Limita-se a apontar o método e detalhar como este foi desenvolvido no contexto do estudo proposto, preterindo detalhes de seu funcionamento interno.

Para a seleção de atributos foram adotadas estratégias usadas por demais autores correlacionando a classe farmacêutica aos atributos apropriados como no estudo realizado por Cano e Fujita (1988), que selecionaram atributos como espectro de ação, farmacocinética, reações adversas, estabilidade e custo diário de tratamento. Em estudo realizado para seleção de heparinas no hospital público (AMARAL e PROVIN, 2011) utilizaram os atributos: efetividade, segurança, experiência com o fármaco, comodidade do fármaco e custo.

Para a aplicação do método AHP foi definido o objetivo: "escolher o melhor fármaco betabloqueador para tratamento de hipertensão". Decompôs-se o problema em hierarquias sistemáticas, optou-se pela seleção de uma alternativa à luz dos critérios:

○ Efetividade;

- Custo;

- Experiência com o Fármaco;

○ Segurança.

Para o critério segurança foram estabelecidos subcritérios relacionados aos efeitos colaterais dos betabloqueadores:

- Agravamento da Insuficiência Cardíaca Congestiva (ICC);

- Alterações Metabólicas;

- Bradicardia;

○ Brocoespasmo;

- Agravamento da Doença Arterial Periférica (DAP).

As alternativas possíveis foram selecionados cinco fármacos betabloqueadores mais utilizados em hipertensão arterial, segundo entrevista prévia com especialistas:

○ Atenolol;

- Carvedilol;

- Metropolol;

- Propranolol;

- Sotalol.

Os pesos conferidos aos critérios de seleção foram definidos junto a comunidade médica através da aplicação de um questionário a sete profissionais especialistas em cardiologia no município de Itaperuna, RJ. O critério efetividade foi considerado o mais importante, seguido do critério segurança, custo e experiência com o fármaco. No critério segurança, o subcritério broncoespasmo foi considerado o mais grave seguido do agravamento da insuficiência cardíaca congestiva (ICC). Agravamento da doença arterial periférica (DAP) e alterações metabólicas foram consideradas menos graves (Figura 2). 


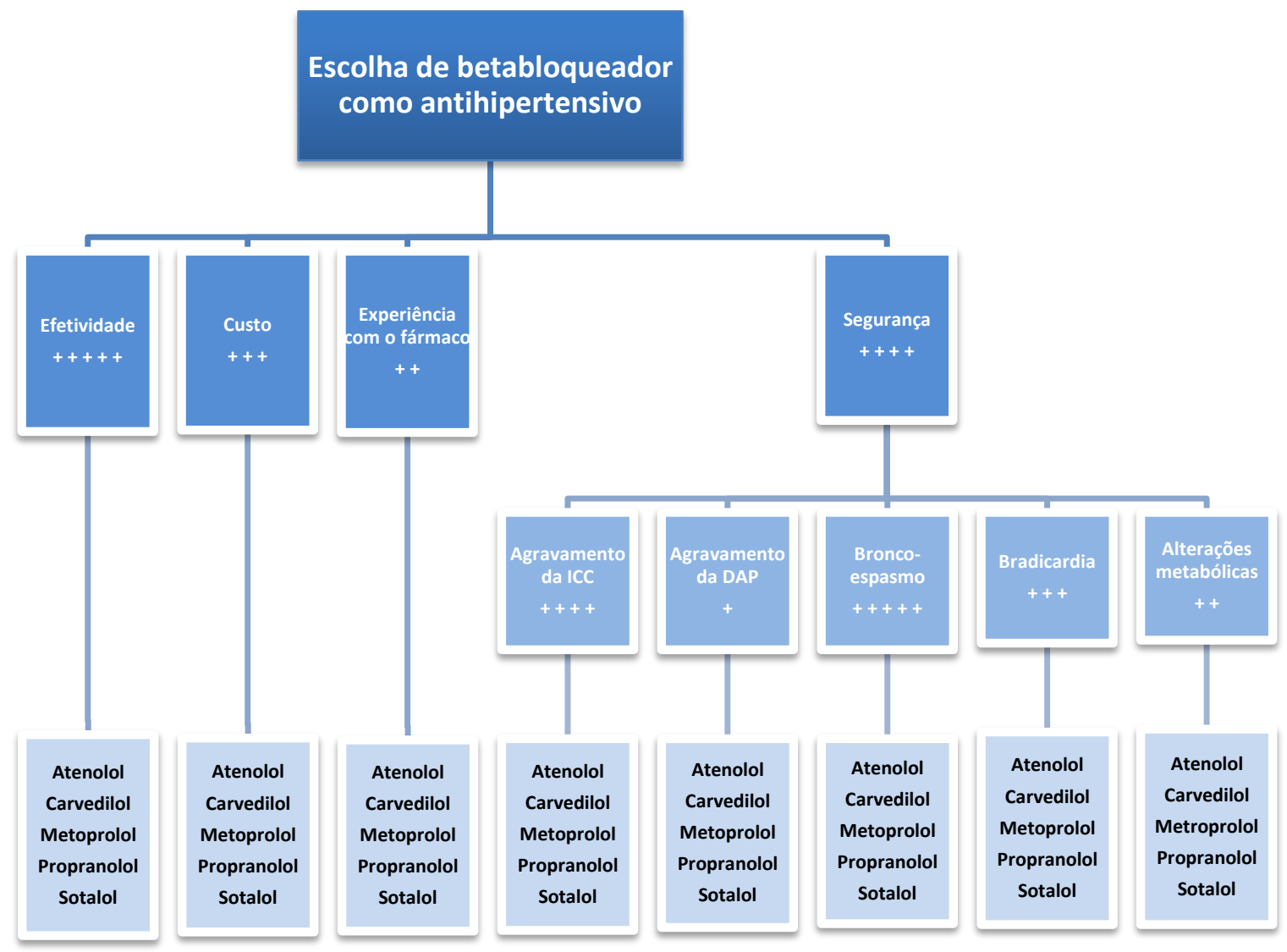

Figura 2: Estrutura hierárquica usada na tomada de decisão para aquisição de betabloqueadores adrenérgicos. A caixa superior (em azul escuro) mostra o objetivo da proposta, em segundo nível são apresentados os critérios e subcritérios e no último nível as alternativas. Os símbolos + indicam o peso dos critérios e subcritérios usados à luz das alternativas propostas.

Para estabelecer as comparações das alternativas, par a par, à luz dos critérios e subcritérios foram realizados levantamentos bibliográficos sobre a efetividade dos fármacos estudados, o custo dos mesmos junto ao balcão de preços em saúde e aplicação de um questionário estruturado aplicado a um grupo de cardiologistas (Quadro 4).

Quadro 4: Critérios usados na seleção e forma de obtenção dos dados.

\begin{tabular}{|l|l|}
\hline CRITÉRIOS & FONTE DAS INFORMAÇÕES \\
\hline Efetividade & Questionários aplicados aos especialistas (cardiologistas). \\
\hline Custo & Banco de Preços em Saúde. \\
\hline Experiência com o fármaco & Número de publicações com o fármaco nos últimos 10 anos, no portal Pubmed. \\
\hline Segurança & $\begin{array}{l}\text { Serão avaliados os sub-critérios como efeitos adversos: broncoespasmo, } \\
\text { agravamento de ICC, agravamento da doença arterial periférica (DAP), } \\
\text { bradicardia e alterações metabólicas. }\end{array}$ \\
\hline
\end{tabular}

$\mathrm{Na}$ análise de custo, os fármacos foram submetidos à pesquisa de preços através do Banco de Preços em Saúde (BPS), um sistema informatizado que registra, armazena e disponibiliza, por meio da internet, os preços de medicamentos e produtos para a saúde, que são adquiridos por instituições públicas e privadas cadastradas no sistema (BRASIL, 2013). Os preços são inseridos pelas próprias instituições e representam os valores pagos no momento da compra daqueles bens. Além de tornar públicas as informações sobre os preços, o sistema proporciona a visualização de relatórios gerenciais, visando auxiliar a instituição na gestão de seus recursos financeiros.

Para o parâmetro experiência com o fármaco foi considerada a variável quantidade de publicações citadas no PubMed, que os cinco anti-hipertensivos analisados obtiveram em 10 


\section{Biomedica Brasiliensia}

anos (outubro/2001 a setembro/2011). Para tanto, selecionou-se todos aqueles artigos que apareceram, em qualquer campo, o nome genérico dos agentes medicamentos analisados (Tabela $3)$.

Para a avaliação dos subcritérios no atributo segurança os efeitos adversos considerados mais graves são avaliados de acordo com a bibliografia, além da experiência de sete especialistas em cardiologia, que tiveram suas opiniões obtidas através de um questionário fechado de caráter descritivo, no qual especialistas ranqueiam, pelo método Borda, com notas de $0,1,2,3$ e 4 de acordo com sua observação pessoal para os efeitos de broncoespasmo, bradicardia, alterações metabólicas, agravamento da insuficiência cardíaca congestiva (ICC) e agravamento da doença arterial periférica (DAP) para cada fármaco estipulado como alternativas, onde a melhor opção, com menos efeitos colaterais recebeu as maiores notas. Da mesma forma esses efeitos foram ranqueados segundo seu grau de importância, os mais significativos, que apresentam maiores problemas ao paciente.

Para o critério efetividade as maiores notas do método Borda foram dadas aos fármacos mais efetivos, por exemplo, o especialista dá a nota máxima àquela que considera a melhor opção, e notas menores em ordem decrescente às outras opções de acordo com o seu critério de escolha. Após todos os especialistas estabelecerem suas notas elas são somadas e a opção que obteve maior nota é considerada a melhor opção.

Para os subcritérios as preferência foram invertidas. Onde se lê preferência por atenolol em relaçao ao carvedilol, lê-se preferência por carvedilol, sem alterar os julgamentos. Os resultados obtidos foram os mesmos.

\section{Resultados}

Os preços dos fármacos e os estados brasileiros que os compraram estão relacionados na Tabela 2. Pose-se observar que o Atenolol e o Propanolol são os fármacos de menor custo e adquiridos na maioria dos estados brasileiros. Metoprolol succinato e Sotalol cloridrato apresentam maior custo e adquiridos em poucos estados da federação.

Tabela 2: Preços mínimos, máximos e médios dos medicamentos e estados brasileiros onde são adquiridos.

\begin{tabular}{|c|c|c|c|c|}
\hline Medicamento & $\begin{array}{c}\text { Preço } \\
\text { máximo }\end{array}$ & $\begin{array}{c}\text { Preço } \\
\text { mínimo }\end{array}$ & $\begin{array}{l}\text { Preço } \\
\text { médio }\end{array}$ & Itens levantados em \\
\hline Atenolol 50mg genérico & 0,1100 & 0,0097 & 0,0139 & $\begin{array}{c}\text { AL/CE/MG/MT/PI/PR/RJ/ } \\
\text { RS/SE/SP }\end{array}$ \\
\hline Carvedilol 3,125mg genérico & 0,2600 & 0,0385 & 0,0585 & $\begin{array}{c}\mathrm{AC} / \mathrm{AL} / \mathrm{MG} / \mathrm{MT} / \mathrm{PE} / \mathrm{PI} / \mathrm{PR} / \mathrm{RJ} / \\
\mathrm{SE} / \mathrm{SP} / \mathrm{TO}\end{array}$ \\
\hline Metoprolol succinato $50 \mathrm{mg}$ & 1,1000 & 0,7700 & 0,8322 & $\mathrm{CE} / \mathrm{ES} / \mathrm{MG} / \mathrm{PI} / \mathrm{PR} / \mathrm{RJ} / \mathrm{SP}$ \\
\hline $\begin{array}{l}\text { Propranolol cloridrato } 40 \mathrm{mg} \\
\text { genérico }\end{array}$ & 0,0700 & 0,0090 & 0,0112 & $\begin{array}{c}\mathrm{AL} / \mathrm{BA} / \mathrm{CE} / \mathrm{DF} / \mathrm{MG} / \mathrm{PR} / \mathrm{RJ} / \\
\mathrm{RS} / \mathrm{SE} / \mathrm{SP} / \mathrm{TO}\end{array}$ \\
\hline Sotalol cloridrato $120 \mathrm{mg}$ & 0,9700 & 0,8450 & 0,9064 & RJ \\
\hline
\end{tabular}

Para a análise de efetividade foram analisados os fármacos comparados em relação a estudos com a redução de hipertensão arterial, tais observações são descritas na Tabela 3 . O Atenolol foi considerado o fármaco mais efetivo, média das notas 3,7, enquanto que o Sotalol foi considerado o menos efetivo, 1,0 , onde a nota máxima seria 4,0 .

Tabela 3: Efetividade dos fármacos quanto ao efeito anti-hipertensivo.

\begin{tabular}{lccc}
\hline Fármaco & Borda & Média das notas & Ranking \\
\hline Atenolol & 26 & 3,7 & $1^{\mathbf{o}}$ \\
Carvedilol & 12 & 1,7 & $3^{\mathbf{o}}$ \\
Metropolol & 15 & 2,1 & $2^{\mathbf{o}}$ \\
Propanolol & 10 & 1,4 & $4^{\mathbf{o}}$ \\
Sotalol & 7 & 1,0 & $5^{\text {o }}$ \\
\hline
\end{tabular}


Na tabela 4 observam-se os resultados obtidos relativos à experiência com os fármacos estudados. O Propanolol é o fármaco mais estudado, com mais de $40 \%$ das publicações encontradas. Os fármacos Atenolol e Metoprolol tem cada um, cerca de $20 \%$ das publicações, enquanto que o Sotalol é o menos estudado.

Tabela 4: Quantidade de publicações sobre betabloqueadores localizados na base Pubmed entre 2001 e 2011.

\begin{tabular}{|c|c|c|}
\hline Medicamento & Número de publicações & Proporção \\
\hline Atenolol & 2.021 & $19,01 \%$ \\
\hline Carvedilol & 1.531 & $14,40 \%$ \\
\hline Metoprolol & 1.949 & $18,34 \%$ \\
\hline Til2016Propranolol & 4.413 & $41,51 \%$ \\
\hline Sotalol & 716 & $6,74 \%$ \\
\hline TOTAL & 10.674 & $100,0 \%$ \\
\hline
\end{tabular}

No atributo segurança, a tabela 5 apresenta os resultados obtidos pelo método Borda e as médias das notas atribuídas nos questionários. Segundo os cardiologistas entrevistados, o broncoespasmo é o efeito colateral mais comum e perigoso à saúde dos pacientes $(3,3)$. O Agravamento da ICC $(2,9)$ e bradicardia são sintomas colaterais preocupantes $(2,1)$, enquanto que o agravamento da DAP $(0,7)$ e alterações metabólicas $(1,0)$ foram consideradas menos graves.

Tabela 5: Notas e ranking dos efeitos colaterais causados pelo uso de betabloqueadores.

\begin{tabular}{lccc}
\hline Sub-atributo & Borda & Média & Ranking \\
\hline Agravamento da ICC & 20 & 2,9 & $2 .^{\circ}$ \\
Alterações metabólicas & 7 & 1,0 & $4 .^{\circ}$ \\
Bradicardia & 15 & 2,1 & $3 .^{\circ}$ \\
Broncoespasmo & 23 & 3,3 & $1 .^{\text {o }}$ \\
Agravamento da DAP & 5 & 0,7 & $5 .^{\circ}$ \\
\hline
\end{tabular}

Na Tabela 6, Tabela 7 e na Tabela 8 pode-se observar que o Atenolol e o Propanolol são os fármacos mais preocupantes no agravamento da insuficiência cardíaca congestiva, nas alterações metabólicas e bradicardia. Estes mesmos sintomas são pouco evidentes nos pacientes tratados com Carvedilol, considerado o fármaco mais seguro para estes efeitos colaterais.

Tabela 6: Subcritério de segurança, fármaco com maior risco de agravamento da insuficiência cardíaca congestiva (ICC).

\begin{tabular}{lccc}
\hline Medicamento & Borda & Média & Ranking \\
\hline Atenolol & 19 & 2,7 & $2 .^{\circ}$ \\
Carvedilol & 4 & 0,6 & $5 .^{\circ}$ \\
Metoprolol & 10 & 1,4 & $4 .^{\circ}$ \\
Propranolol & 21 & 3,0 & $1 .^{\circ}$ \\
Sotalol & 16 & 2,3 & $3 .^{\circ}$ \\
\hline
\end{tabular}

Tabela 7: Subcritério de segurança, fármaco com maior risco de surgimento de alterações metabólicas.

\begin{tabular}{lccc}
\hline Medicamento & Borda & Média & Ranking \\
\hline Atenolol & 19 & 2,7 & $2 .^{\circ}$ \\
Carvedilol & 7 & 1,0 & $5 .^{\circ}$ \\
Metoprolol & 13 & 1,9 & $3 .^{\circ}$ \\
Propranolol & 20 & 2,9 & $1 .^{\circ}$ \\
Sotalol & 11 & 1,6 & $4 .^{\circ}$ \\
\hline
\end{tabular}


Tabela 8: Subcritério de segurança, fármaco com maior risco de bradicardia.

\begin{tabular}{lccc}
\hline Medicamento & Borda & Média & Ranking \\
\hline Atenolol & 20 & 2,9 & $1 .^{\mathbf{o}}$ \\
Carvedilol & 4 & 0,6 & $5 .^{\circ}$ \\
Metoprolol & 14 & 2,0 & $3 .^{\circ}$ \\
Propranolol & 18 & 2,6 & $2 .^{\circ}$ \\
Sotalol & 14 & 2,0 & $3 .^{\circ}$ \\
\hline
\end{tabular}

O Carvedilol também é seguro para prevenir broncoespasmo e agravamento da pressão arterial periférica (DAP) (tabela 9 e Tabela 10). O Propanolol e Atenolol apresentam riscos para estes efeitos colaterais.

Tabela 9: Subcritério de segurança, fármaco com maior risco de episódios de broncoespasmos.

\begin{tabular}{lccc}
\hline Medicamento & Borda & Média & Ranking \\
\hline Atenolol & 13 & 1,9 & $3^{\mathbf{o}}$ \\
Carvedilol & 4 & 0,6 & $5^{\mathbf{o}}$ \\
Metoprolol & 16 & 2,3 & $2^{\mathbf{o}}$ \\
Propranolol & 24 & 3,4 & $1^{\mathbf{0}}$ \\
Sotalol & 13 & 1,9 & $3^{\mathbf{o}}$ \\
\hline
\end{tabular}

Tabela 10: Subcritério de segurança, fármaco com maior risco de agravamento da doença arterial periférica (DAP).

\begin{tabular}{lccc}
\hline Medicamento & Borda & Média & Ranking \\
\hline Atenolol & 18 & 2,6 & $2^{\mathbf{o}}$ \\
Carvedilol & 7 & 1,0 & $4^{\text {o }}$ \\
Metoprolol & 13 & 1,9 & $3^{\text {o }}$ \\
Propranolol & 24 & 3,4 & $1^{\text {o }}$ \\
Sotalol & 8 & 1,1 & $5^{\text {o }}$ \\
\hline
\end{tabular}

O mecanismo interno do método AHP permite que as avaliações cometam até $10 \%(0,01)$ de inconsistência, admitindo este valor como normal em julgamentos humanos (Costa, 2006; Saaty, 1977). A tabela 11 mostra os IC obtidos nos julgamentos dos critérios e subcritérios.

Tabela 11: Razão de consistência (RC) dos julgamentos obtidos na pesquisa.

\begin{tabular}{lcc}
\hline Critério & Subcritério & RC \\
\hline Bloqueadores para hipertensão & - & 0,086 \\
Efetividade & - & 0,085 \\
Custo & - & 0,089 \\
Experiência com o fármaco & - & 0,051 \\
Segurança & - & 0,054 \\
& Agravamento da ICC & 0,074 \\
& Broncoespasmo & 0,062 \\
Segurança & Bradicardia & 0,034 \\
& Alterações metabólicas & 0,038 \\
& Agravamento da DAP & 0,058 \\
\hline
\end{tabular}

Para avaliação dos resultados encontrados com o método proposto foi realizada uma primeira estimativa dos valores da importância correspondente aos critérios escolhidos. A Tabela 12 mostra a matriz de comparação paritária para a determinação da importância relativa atribuída aos critérios, seguindo-se da Figura 3 que apresenta os índices relativos à importância dos mesmos. 
Tabela 12: Matriz de comparação paritária entre os critérios.

\begin{tabular}{lcccc}
\hline & Efetividade & Custo & $\begin{array}{c}\text { Experiência } \\
\text { com o } \\
\text { fármaco }\end{array}$ & Segurança \\
\hline $\begin{array}{l}\text { Efetividade } \\
\text { Custo }\end{array}$ & 1 & 3 & 5 & 2 \\
$\begin{array}{l}\text { Experiência com o } \\
\text { fármaco }\end{array}$ & $1 / 3$ & 1 & 4 & $1 / 4$ \\
Segurança & $1 / 5$ & $1 / 4$ & 1 & $1 / 5$ \\
\hline & $1 / 2$ & 4 & 5 & 1 \\
\hline
\end{tabular}

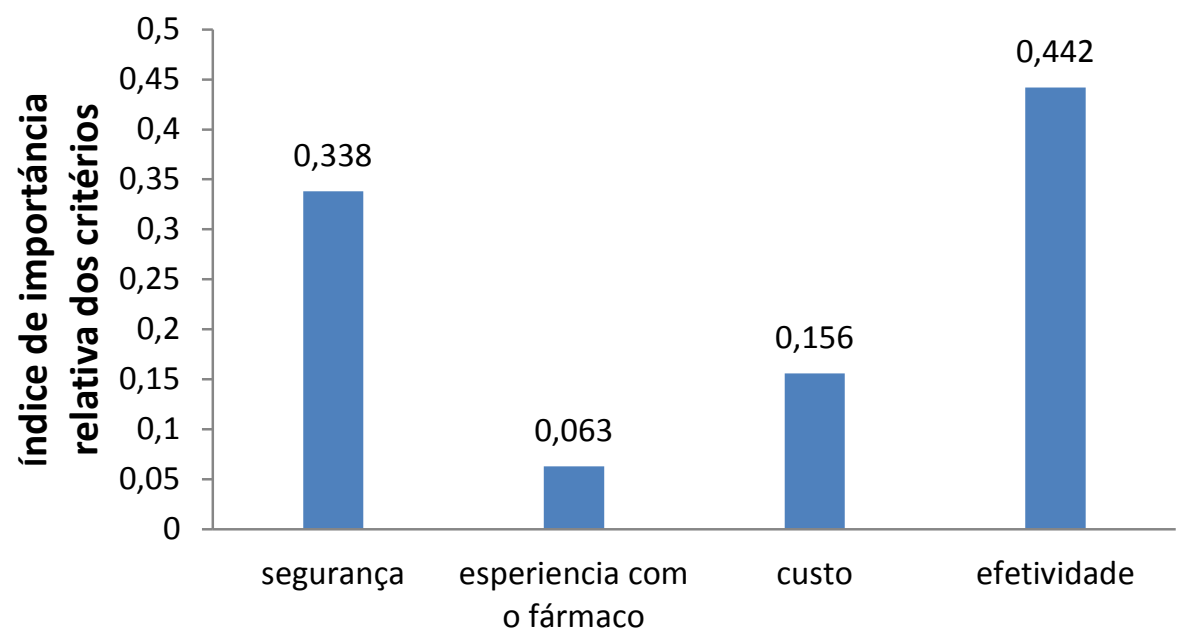

\section{Critérios}

Figura 3: Índice de importância relativa aos critérios.

A

Figura 4 apresenta o resultado final pelo método AHP, que sugere a aquisição do Atenolol pelo poder público.

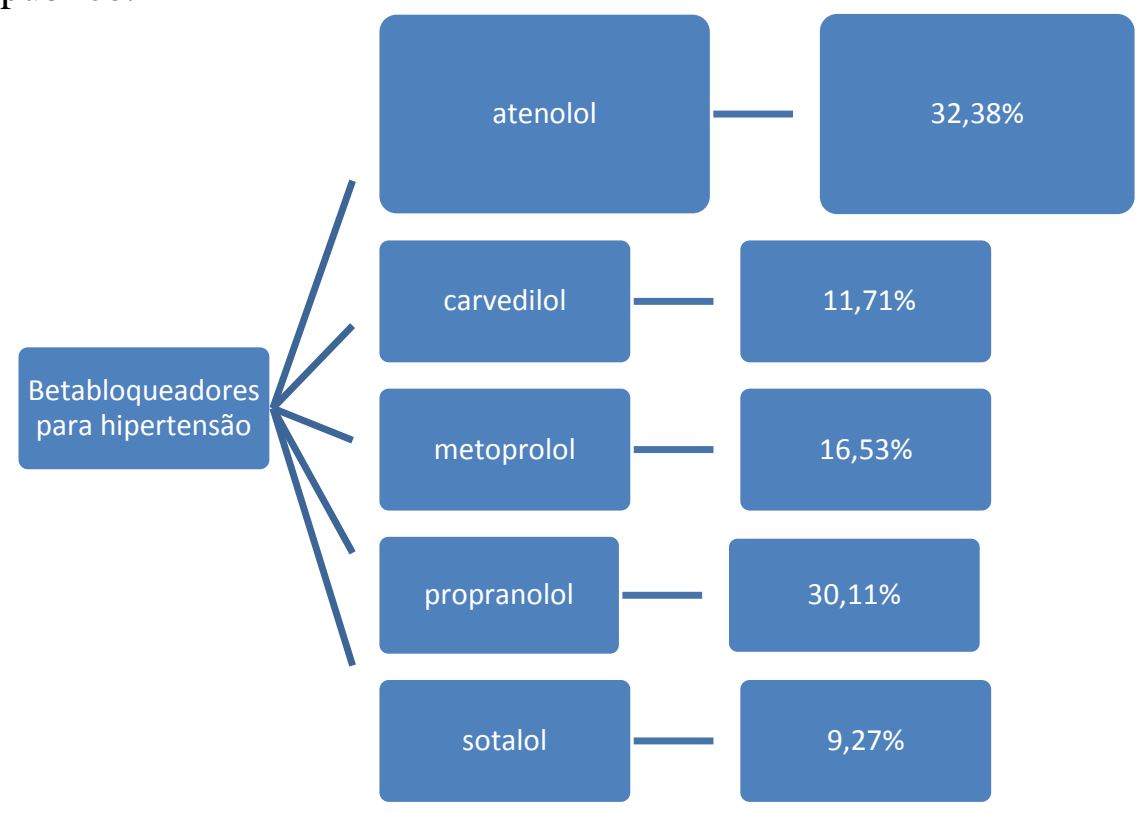

Figura 4: Resultado final do problema proposto. O maior percentual indica o resultado sugerido pelo método AHP. 


\section{Discussão}

A fase de avaliação do problema está dividida, segundo o método AHP, na determinação da importância relativa dos critérios, na determinação do nível de preferência das alternativas e valoração global das alternativas (ABREU et al., 2000).

Utilizando-se a metodologia de decisão multicritério AHP, o betabloqueador que se apresentou como melhor escolha foi o atenolol (32,38\%), figura 3, em consonância com a escolha dos especialistas entrevistados e resultado obtido pelo método Borda.

O propranolol apresenta um maior número de trabalhos publicados $(41,51 \%)$ por ser o fármaco protótipo, lançado na Europa em 1965, após pesquisas realizadas pelo britânico Sir James Whyte Black na década de 50. Os demais fármacos foram desenvolvidos através de modificações na molécula de propranolol, com o intuito de fazer um melhoramento molecular (BARREIRO e FRAGA, 2008).

Em relação ao custo dos medicamentos o propranolol seria a melhor escolha, pois seu preço médio ( $\mathrm{R} \$ 0,0112$ /unidade) é $19,42 \%$ mais barato que o atenolol ( $\mathrm{R} \$ 0,0139$ ), enquanto que a diferença para o sotalol que é cerca de $800 \%$ mais caro. O propranolol, o atenolol e o carvedilol possuem preços baixos, devido ao fato de estarem no mercado a mais tempo e suas patentes já estarem expiradas faz com que a livre concorrência para a produção e comercialização dos produtos torne-os mais acessíveis.

No critério segurança, dos cinco subcritérios relacionados, broncoespasmo foi considerado pelos profissionais médicos entrevistados como sendo o mais relevante (média 3,3), pois se mostra como um fator de risco muito alto para pacientes que possuam alguma co-morbidade pulmonar, além da diminuição da adesão à terapia devido às recorrentes crises de tosse seca. quando se leva em consideração a segurança do fármaco, o carvedilol seria o que apresentaria menos efeitos colaterais, seguindo-se pelo sotalol, metoprolol, atenolol e propranolol, este apresenta maiores riscos no seu uso em quatro dos cinco sub-critérios analisados pelos especialistas.

Em relação aos outros subcritérios, o medicamento atenolol não é considerado um dos mais seguros, sendo o que causa incidência de bradicardia e estando em segundo no restante dos subcritérios, agravamento da ICC e da DAP e alterações metabólicas. Porém esses subcritérios têm menor peso em relação ao broncoespasmo.

O atenolol é o fármaco com maior efetividade para o tratamento de hipertensão, segundo as respostas dos especialistas (3,7), seguindo-se pelo metoprolol $(2,1)$. Estes dois medicamentos betabloqueadores que apresentam maior seletividade por seus receptores (PAGE et al., 2004).

\section{Considerações Finais}

A estrutura da matriz de decisão proposta permitiu a resolução do problema e pode ser utilizada por gestores da área da saúde em futuras decisões. A estrutura proposta buscou uma solução com base no custo-efetividade, para escolher um anti-hipertensivo que apresente poucos efeitos colaterais, o que resultaria, por parte do paciente, baixa qualidade de vida e retorno aos centros de saúde e uso de outros medicamentos no combate aos efeitos colaterais, o que resultaria em outros custos. Executando-se esse processo, o decisor analisa o problema, identifica o problema de decisão correto que precisa ser solucionado.

Todas as drogas para tratamento de hipertensão possuem efeitos colaterais. A escolha das drogas é influenciada pela demonstração dos efeitos benéficos, custos, tolerabilidade em pacientes e distúrbios concomitantes (PAGE et al., 2004).

Pelo método de Borda, os especialistas consideraram o atenolol como o fármaco mais efetivo (26 pontos). Quanto ao critério de segurança, os especialistas elegeram o broncoespasmo 
(23 pontos) como sendo o item o efeito colateral mais relevante na hora de prescreve o medicamento, sendo o propranolol o que apresenta a maior incidência desse efeito.

O propranolol é o fármaco com menor custo (R \$ 0,0112) (BRASIL, 2013) e também o com maior número de publicações no banco de dados da Pubmed.com (4.413 publicações). $\mathrm{O}$ atenolol se mostrou como a melhor opção $(32,38 \%)$ levando-se em consideração os critérios estabelecidos. Confirmando os dados obtidos com os especialistas que o consideram mais efetivo e baixos problemas quanto à sua segurança através do método Borda.

O método AHP mostrou-se como uma opção para o apoio a tomada de decisão para principalmente onde há padronização de determinados medicamentos dentro de uma classe medicamentosa como hospitais e secretarias de saúde de municípios e estados, para que possam adquirir fármacos utilizando análises complexas.

\section{Referências}

ABREU, L. M. DE et al. Escolha de um programa de controle da qualidade da água para consumo humano: aplicação do Método AHP. Revista Brasileira de Engenharia Agrícola e Ambiental, v. 4, n. 2, p. 257-262, 2000.

AMARAL, M. F.; PROVIN, M. P. Aplicabilidade da metodologia multiatributo na seleção de heparinas em hospital público. Revista Eletrônica de Farmácia, v. 7, n. 4, p. 13, 16 fev. 2011.

AZAMBUJA, M. I. R. et al. Impacto econômico dos casos de doença cardiovascular grave no Brasil: uma estimativa baseada em dados secundários. Arquivos Brasileiros de Cardiologia, v. 91, n. 3, p. 163-171, set. 2008.

BARREIRO, E. J.; FRAGA, C. A. M. Química Medicinal: As Bases Moleculares da Ação dos Fármacos. 2. ed. Porto Alegre: Artmed, 2008.

BORTOLOTTO, L. A.; CONSOLIM-COLOMBO, F. M. Betabloqueadores adrenérgicos. Rev Bras Hipertens, v. 16, n. 4, p. 215-20, 2009.

BRASIL, M. DA S. Banco de Preços em Saúde. Institucional. Disponível em: <http://portalsaude.saude.gov.br/index.php/cidadao/principal/banco-de-precos-em-saude>.

Acesso em: 23 abr. 2016.

BRUNTON, L.; LAZO, J. S.; PARKER, K. L. As bases farmacológicas da terapêutica. [s.l: s.n.].

CAMPOS, F. V. DE S.; PORTO, L. G. G. Qualidade de vida e nível de atividade física de pacientes em fase ambulatorial da reabilitação cardíaca. Revista Brasileira de Atividade Física \& Saúde, v. 14, n. 2, p. 86-95, 10 set. 2012.

CANO, S. B.; FUJITA, N. K. Formulary evaluation of third-generation cephalosporins using decision analysis. American Journal of Hospital Pharmacy, v. 45, n. 3, p. 566-569, mar. 1988.

CORDEIRO, B. C.; LEITE, S. N. O farmacêutico na atenção à saúde. 2. ed. Itajaí: UNIVALI, 2005.

COSTA, H. G. Estruturas de Suporte à Decisão. Niterói: UFF, 2005.

Auxílio multicritério à decisão: método AHP. Rio de Janeiro: Abepro, 2006. 
GOMES, L. F. A. M.; GOMES, C. F. S. Tomada de decisão gerencial: enfoque multicritério. 4. ed. São Paulo: Atlas, 2012.

GOMES, M. A. M. et al. IV Diretrizes Brasileiras de Hipertensão Arterial Grupos de trabalho. Arquivos Brasileiros de Cardiologia, v. 82, mar. 2004.

HELFAND, M. et al. Drug Class Review: Beta Adrenergic Blockers: Final Report Update 4. Portland (OR): Oregon Health \& Science University, 2009.

KOROLKOVAS, A.; FRANÇA, F. F. DE A. C. DE. Dicionário Terapêutico Guanabara. 21. ed. Rio de Janeiro: Grupo Gen - Guanabara Koogan, 2009.

LIBERATORE, M. J.; NYDICK, R. L. The analytic hierarchy process in medical and health care decision making: A literature review. European Journal of Operational Research, v. 189, n. 1, p. 194-207, 16 ago. 2008.

MENDES, L. F. R.; ERTHAL JÚNIOR, M. Estudo bibliométrico sobre o método de Análise Hierárquica (AHP): 1979 a 2012. In: XXXII ENCONTRO NACIONAL DE ENGENHARIA DE PRODUÇÃO. Bento Gonçalves: ABEPRO, 2012

PAGE, C. P. et al. Farmacologia integrada. Traducao Ida Cristina Gubert. Barueri, SP: Manole, 2004.

PIOLA, S. F.; VIANNA, S. M. Saúde no Brasil: algumas questões sobre os sistema único de saúde (SUS). [s.l.] CEPAL/IPEA, 2009. Disponível em: <http://repositorio.cepal.org/bitstream/handle/11362/1349/LCbrsR200_pt.pdf;jsessionid=740059 DA2C36FDEC6813B59585B30816? sequence=1>. Acesso em: 21 abr. 2016.

PUCCI, N. et al. Conhecimento sobre hipertensão arterial sistêmica e adesão ao tratamento antihipertensivo em idosos. Rev Bras Cardiol, v. 25, n. 4, p. 322-9, 2012.

RANG, H. P. Rang \& Dale farmacologia. Rio de Janeiro: Elsevier, 2012.

RASCATI, K. L. Introdução à Farmacoeconomia. Porto Alegre: Artmed, 2009.

SAATY, T. L. A scaling method for priorities in hierarchical structures. Journal of Mathematical Psychology, v. 15, n. 3, p. 234-281, jun. 1977.

SECOLI, S. R. et al. Pharmacoeconomics: resultant perspective of decisions process. Ciência \&amp; Saúde Coletiva, v. 10, p. 287-296, dez. 2005.

SILVA, C. A. S. DA; FERREIRA, C. E. S. Efeitos de um programa de reabilitação cardíaca na qualidade de vida relacionada à saúde (SF-36). Educação Física em Revista, v. 5, n. 1, 2011.

TONON, L. M.; TOMO, T. T.; SECOLI, S. R. Farmacoeconomia: análise de uma perspectiva inovadora na prática clínica da enfermeira. Texto \&amp; Contexto - Enfermagem, v. 17, n. 1, p. 177-182, mar. 2008. 
VANZELLI, A. S. et al. Associação de betabloqueadores e treinamento físico na insuficiência cardíaca de camundongos. Arquivos Brasileiros de Cardiologia, v. 95, n. 3, p. 373-380, set. 2010.

VIEIRA, F. S. Gasto do Ministério da Saúde com medicamentos: tendência dos programas de 2002 a 2007. Revista de Saúde Pública, v. 43, n. 4, p. 674-681, ago. 2009.

VOSGERAU, M. Z. DA S.; CABRERA, M. A. S.; SOUZA, R. K. T. Saúde da família e utilização de medicamentos anti-Hipertensivos e antidiabéticos. Rev Bras Cardiol, v. 24, n. 2, p. 95-104, 2011.

WANNMACHER, L. Análise dos estoques domiciliares de medicamentos essenciais no sul do Brasil: Uso racional de medicamentos: temas selecionados. Brasília: Organização PanAmericana da Saúde / Organização Mundial da Saúde, 2007. Disponível em: <Organização PanAmericana da Saúde/>. Acesso em: 23 abr. 2016.

WHO. Essential medicines. Institucional. Disponível em: <http://www.who.int/topics/essential_medicines/en/>. Acesso em: 23 abr. 2016. 